Article

\title{
Universal Thermodynamics in the Context of Dynamical Black Hole
}

\author{
Sudipto Bhattacharjee and Subenoy Chakraborty * \\ Department of Mathematics, Jadavpur University, Kolkata 700032, West Bengal, India; slg00sudipto@gmail.com \\ * Correspondence: schakraborty.math@gmail.com
}

Received: 27 April 2018; Accepted: 22 June 2018; Published: 1 July 2018

check for updates

\begin{abstract}
The present work is a brief review of the development of dynamical black holes from the geometric point view. Furthermore, in this context, universal thermodynamics in the FLRW model has been analyzed using the notion of the Kodama vector. Finally, some general conclusions have been drawn.
\end{abstract}

Keywords: dynamical black hole; trapped surfaces; universal thermodynamics; unified first law

\section{Introduction}

A black hole is a region of space-time from which all future directed null geodesics fail to reach the future null infinity $I^{+}$. More specifically, the black hole region $B$ of the space-time manifold $M$ is the set of all events $P$ that do not belong to the causal past of future null infinity, i.e.,

$$
B=M-J^{-}\left(I^{+}\right) .
$$

Here, $J^{-}\left(I^{+}\right)$denotes the causal past of $I^{+}$, i.e., it is the set of all points that causally precede $I^{+}$. The boundary of the black hole region is termed as the event horizon $(H)$,

$$
H=\partial B=\partial\left(J^{-}\left(I^{+}\right)\right)
$$

A cross-section of the horizon is a 2D surface $H(\Sigma)$ obtained by intersecting the event horizon with a space-like hypersurface $\Sigma$. As event the horizon is a causal boundary, it must be a null hypersurface generated by null geodesics that have no future end points.

In the black hole region, there are trapped surfaces that are closed 2-surfaces $(S)$ such that both ingoing and outgoing congruences of null geodesics are orthogonal to $S$, and the expansion scalar is negative everywhere on $S$. Therefore, the 3D boundary of the space-time region that contains trapped surfaces is the trapping horizon. Further, a closed two-surface on which one of the congruences has zero expansion is termed as a marginally-trapped surface or apparent horizon. For a stationary black hole, the apparent horizon coincides with the event horizon, while in the dynamical situation, the apparent horizon always lies inside the black hole region unless the null energy condition (NEC) is violated.

According to Hawking and Penrose, the existence of trapped surfaces inside a black hole implies the formation of a singularity provided the strong energy condition (SEC) is violated [1]. Geometrically, the existence of singularity implies geodesic (time-like or null) incompleteness.

A stationary black hole that is static must be spherically symmetric [2-6]. On the other hand, the stationary space-time of a rotating black hole is necessarily axially symmetric, and it admits two Killing vectors $t^{\alpha}$ and $\phi^{\alpha}$, i.e., the Lie derivatives of the metric tensor along the vectors vanish, more specifically $\mathcal{L}_{t} g^{\alpha \beta}=0$ and $\mathcal{L}_{\phi} g^{\alpha \beta}=0$, respectively. Then, the linear combination $\xi^{\alpha}=t^{\alpha}+\Omega_{H} \phi^{\alpha}$ 
is null on the event horizon ( $\Omega_{H}$ is the black hole's angular velocity). In fact, $\xi^{\alpha}$ is tangent to the horizon's null generators. The surface gravity $\kappa$ of the black hole is defined by the relation [7]:

$$
\xi_{; \beta}^{\alpha} \xi^{\beta}=\kappa \xi^{\alpha}
$$

on the horizon. Note that $\kappa$ is constant along the horizon's null generators, i.e., uniform over the entire horizon.

Now, we shall consider a congruence of time-like geodesics with four velocity $u^{\alpha}$. The divergence of the four velocity is called the expansion scalar, i.e., $\theta=u_{; \alpha}^{\alpha}$. The rotation tensor is given by:

$$
\omega_{\alpha \beta}=u_{[\alpha ; \beta]}-\dot{u}_{[\alpha} u_{\beta]},
$$

where $u_{[\alpha ; \beta]}=\frac{1}{2}\left(u_{\alpha ; \beta}-u_{\beta ; \alpha}\right)$ and $\dot{u}_{[\alpha} u_{\beta]}=\frac{1}{2}\left(\dot{u}_{\alpha} u_{\beta}-\dot{u}_{\beta} u_{\alpha}\right)$. The shear tensor is given by $\sigma_{\alpha \beta}=$ $u_{(\alpha ; \beta)}-\frac{1}{3} \theta h_{\alpha \beta}-\dot{u}_{(\alpha} u_{\beta)}$ where $u_{(\alpha ; \beta)}=\frac{1}{2}\left(u_{\alpha ; \beta}+u_{\beta ; \alpha}\right)$ and $\dot{u}_{(\alpha} u_{\beta)}=\frac{1}{2}\left(\dot{u}_{\alpha} u_{\beta}+\dot{u}_{\beta} u_{\alpha}\right)$. Now, using the facts (i) due to hypersurface orthogonal nature of congruence of null generators, the rotation tensor $\omega_{\alpha \beta} \equiv 0$, and (ii) due to the stationarity of the event horizon, both $\theta$ and $\frac{d \theta}{d \lambda}$ are both $(\lambda$ is the proper time), the Raychaudhuri equation for null geodesics [8,9]:

$$
\frac{\mathrm{d} \theta}{\mathrm{d} \lambda}=-\frac{1}{2} \theta^{2}-\sigma^{\alpha \beta} \sigma_{\alpha \beta}-R_{\alpha \beta} \xi^{\alpha} \xi^{\beta}
$$

implies (using Einstein field equations): (a) $T_{\alpha \beta} \xi^{\alpha} \xi^{\beta}=0$ on the event horizon. Therefore, matter cannot flow across the event horizon, i.e., the generators would get focused and the black hole would not be stationary. (b) $\sigma_{\alpha \beta}=0$, i.e., the null generators of the event horizon have a vanishing shear tensor.

Usually, a black hole is characterized by its event horizon. However, the very definition of event horizon demands that it could never be observed by any means in finite time; its existence can only be located in infinite future. According to Hayward, the event horizon does not have any physical effect [10], and it may pass through an observer at any finite time without any imprint. Consequently, the notion of trapped surfaces may be interesting in the context of a black hole, as they may hint that a black hole has been formed.

In $4 \mathrm{D}$ space-time, let us consider 2D surfaces that are space-like and closed (i.e., topologically, they are two-spheres). Now, at each point on this 2D surface, one may consider two null directions normal to the surface, i.e., there are two distinct future directed families of null geodesics emerging from the surface; along the outer and inner directions. If it is so happens that both the families of null geodesics orthogonal to the surface converge, then the two-surface is said to be trapped. Geometrically, it implies that both the null geodesics (orthogonal to the surface) emitting from every point of the surface will decrease in area. More specifically, these two-surfaces are termed as future trapped surfaces. The convergence of outgoing null geodesics implies that there is a strong gravitational field, tending to focus on the geodesics, and something is unusual due to the presence of a trapped surface.

Let $\zeta$ be a closed space-like two-surface (topologically two-sphere) and contained in a 3D space-like hypersurface $\Sigma$. Suppose $\vec{s}$ is a unit space-like vector normal to $\zeta$, but lying on $\Sigma$. Similarly $\vec{n}$, is a future-directed unit time-like normal field to $\Sigma$. Then, $\overrightarrow{K_{ \pm}}=\vec{n} \pm \vec{s}$ are the outgoing and ingoing future-directed null normal fields with normalization: $\overrightarrow{K_{+}} \overrightarrow{K_{-}}=-2$. Then, the expansion scalars $\theta_{ \pm}$of the congruence of null geodesics having tangent vector fields $\overrightarrow{K_{ \pm}}$are defined as:

$$
\theta_{ \pm}=\gamma^{A B} K_{A B}\left(\overrightarrow{K_{ \pm}}\right) \text {on } \zeta
$$

where $\gamma_{A B}$ is the first fundamental form, i.e., the induced metric on the two-surface $\zeta$ and $K_{A B}$ is the second fundamental form, i.e., extrinsic curvature of the two-surface $\zeta$. The above scalars measure to what extent the congruence of null geodesics expand, namely $\theta>0(<0)$ implies the divergence (convergence) of the congruence of null geodesics. Thus, the trapping nature of $\zeta$ is characterized by the signs of $\theta_{ \pm}$. It should be noted that the values of $\theta_{ \pm}$depend on the choice of the null normals, 
which cannot be uniquely defined; rather, they depend on the choice of the hypersurface $\Sigma$. However, the signs of $\theta_{ \pm}$do not depend on the choice of the null normals $K_{ \pm}$[11].

Thus, the compact spatial two-surface $\zeta$ with future pointing null directions $\overrightarrow{K_{ \pm}}$(normal to $\zeta$ ) is said to be a future (past) trapped surface if $\left.\theta_{ \pm}\right|_{\zeta}<0\left(\left.\theta_{ \pm}\right|_{\zeta}>0\right)$. However, future trapped surfaces are not quite characterized as a black hole. Therefore, one has to consider marginal surfaces that are just trapped to characterize the boundary of a black hole.

A marginal surface is a spatial two-surface $S$ on which one of the null expansions vanishes (say $\left.\theta_{+}\right|_{S}=0$ ). A trapping horizon is the closure $\overline{\mathcal{H}}$ of a three-surface $\mathcal{H}$ foliated by marginal surfaces on which $\left.\theta_{-}\right|_{\mathcal{H}} \neq 0$ and $\left.\mathcal{L}_{-} \theta_{+}\right|_{\mathcal{H}} \neq 0$. The trapping horizon, as well as marginal surfaces are said to be:

- outer if $\left.\mathcal{L}_{-} \theta_{+}\right|_{\mathcal{H}}<0$, inner if $\left.\mathcal{L}_{-} \theta_{+}\right|_{\mathcal{H}}>0$

- future if $\left.\theta_{-}\right|_{\mathcal{H}}<0$, past if $\left.\theta_{-}\right|_{\mathcal{H}}>0$.

Therefore, for a black hole, the most relevant horizon is the future outer trapping horizon having properties:

- $\quad$ The ingoing light rays should be converging, i.e., $\left.\theta_{-}\right|_{\mathcal{H}}<0$.

- The outgoing light rays should be instantaneously parallel on the horizon, i.e., $\left.\theta_{-}\right|_{\mathcal{H}}=0$.

- They are diverging just outside the horizon and converging just inside, i.e., $\left.\mathcal{L}_{-} \theta_{+}\right|_{\mathcal{H}}<0$.

Therefore, the future outer trapping horizon characterizes a quasi-local black hole horizon. Hawking's area theorem, a fundamental feature of a classical black hole bounded by an event horizon, can be extended to a quasi-local black hole. In fact, the future condition $\left.\theta_{-}\right|_{\mathcal{H}}<0$ guarantees the non-decrease of the horizon area [11].

As the geometric structure of null and spatial hypersurfaces is distinct, it is interesting to study the stationary and dynamical regimes of a quasi-local black hole, characterized by a future outer trapping horizon. In stationary regimes, the geometric structures constructed on a null hypersurface that is foliated by closed outer marginally-trapped surfaces is provided by isolated horizons. In fact, different levels of stationarity for a black hole horizon (in a dynamical environment) are characterized by an isolated horizon. It should be noted that an isolated horizon does not interact with its surroundings, i.e., there is no exchange of information with the surroundings. The event horizons of static Schwarzschild and Reissner-Nordström black holes are isolated horizons.

The dynamical horizon characterizes the non-stationary regime of quasi-local black hole. A space-like hypersurface foliated by closed future marginally-trapped surfaces is termed a dynamical horizon.

The Killing horizon, which is purely local in nature, is defined by the vanishing of the norm of a Killing vector field. The null hypersurface, which is the connected component of the set of points where a Killing field is null, is the Killing horizon. Note that a Killing horizon is not necessarily a black hole horizon, and also, it is not an isolated horizon. Subsequently, Hayward [10-13] initiated the study of black hole thermodynamics for dynamical black holes. He introduced a one-form equation known as the unified first law (UFL), and he had shown its equivalence with the Einstein field equations. It was also shown that by projecting the UFL along the tangent to the trapping horizon, the first law of black hole physics can be obtained for the dynamical black hole.

The paper is organized as follows: In Section 2, we discuss some basic ideas about dynamical black holes. Section 3 deals with the thermodynamics in the homogeneous and isotropic FLRW model. The derivation of the Clausius relation from UFL is presented in Section 4. A new definition of the surface gravity is discussed in Section 5. Section 6 is devoted to concluding remarks.

\section{Preliminaries on Dynamical Black Holes}

The discovery of Hawking that the surface gravity determines the effective temperature of a quantum field on a stationary black hole background created a nice analogy between the laws of 
thermodynamics and certain properties of black holes. As a result, one has to take into account black hole dynamics (i.e., dynamically-evolving black holes) instead of black hole statics. Usually, a black hole is characterized by the event horizon for which the global assumption of asymptotic flatness is essential, and this may not be the case for the real universe.

Hayward, in a series of papers [10-13], introduced the notion of a dynamical, quasi-local definition of black holes in terms of trapping horizons, which are hypersurfaces foliated by marginal surfaces. He then derived a second law of black hole dynamics by expressing the increase of the area element along the trapping horizon, and consequently, black hole dynamics was formulated as the dynamics of trapping horizons. Hayward introduced a new definition of surface gravity for non-stationary black holes to formulate a corresponding first law of black hole dynamics. Instead of using the Kodama vector, he introduced the surface gravity similar to the stationary definition with the Killing vector on the Killing horizon. In this work, we have considered the $3+1$ space-time in the framework of Einstein's gravity.

There is another debated issue in general relativity, namely the idea of energy, as there is no well-accepted definition of gravitational energy. However, for spherical symmetry, Misner-Sharp energy has all the relevant physical properties, and it represents active gravitational energy. This energy is defined as [14]:

$$
E=\frac{r}{2}\left(1-\nabla r \cdot \nabla^{\#} r\right),
$$

where \# stands for the contravariant dual (index raising) with respect to the space-time metric $g$ and the dot denotes contraction. Here, the Newtonian gravitational constant is chosen to be unity, and spatial metrics are taken to be positive definite by sign convention. One can construct two invariant quantities from the energy tensor $T$ as:

$$
w=-\frac{1}{2} \operatorname{Tr} . T
$$

and the vector

$$
\psi=T \nabla r+w \nabla^{\#} r,
$$

where the trace is taken over the 2D space normal to the spheres of symmetry. Here, $w$ is termed as the work function, and $\psi$ is called the energy supply vector. Then, the gradient of the Misner-Sharp energy is related to the Einstein equations as:

$$
\nabla E=A \psi^{\natural}+w \nabla V
$$

where $\lfloor$ denotes the covariant dual.

The 2D space normal to the spherical symmetry is usually characterized by one spatial and one temporal direction, which cannot be chosen uniquely. However, using double-null coordinates $\left(\xi^{+}, \xi^{-}\right)$, each symmetric sphere has two preferred normal directions (the null directions) $\frac{\partial}{\partial \xi^{ \pm}}$. The line element for $4 \mathrm{D}$ space-time in the double-null form can be written as:

$$
\mathrm{d} s^{2}=r^{2} \mathrm{~d} \Omega_{2}^{2}-2 e^{-f} \mathrm{~d} \xi^{+} \mathrm{d} \xi^{-},
$$

where $\mathrm{d} \Omega_{2}^{2}$ is the metric on unit two-sphere and $r \geq 0$ and $f$ are a function of the null coordinates. In this coordinate system, the different components of the UFL are given by:

$$
\begin{aligned}
& E=r\left(\frac{1}{2}+e^{+f} \partial_{+} r \partial_{-} r\right), \\
& w=e^{-f} T^{+-} \\
& \psi=T^{++} \partial_{+} r \partial_{+} T T^{--} \partial_{-r} \partial_{-}, \\
& \partial_{ \pm} E=A \psi_{ \pm}+w \partial_{ \pm} \psi=A e^{f^{+-}}\left(T_{+-} \partial_{ \pm} r-T_{ \pm \pm} \partial_{ \pm} r\right),
\end{aligned}
$$

where the following Einstein field equations are used to derive the last equation. 
The explicit form of the Einstein field equations in this double null coordinates is:

$$
\begin{aligned}
& \partial_{ \pm} \partial_{ \pm} r-\partial_{ \pm} \log \left(-g_{+-}\right) \partial_{ \pm} r=-4 \pi r T_{ \pm \pm}, \\
& r \partial_{+} \partial_{-} r+\partial_{+} r \partial_{-} r-\frac{1}{2} g_{+-}=4 \pi r^{2} T_{+-} \\
& r^{2} \partial_{+} \partial_{-}\left(f_{+-}\right)-2\left(\partial_{+} r\right)\left(\partial_{-} r\right)-e^{-f_{+-}}=8 \pi r^{2}\left(-e^{-f_{+-}}-T_{\theta}^{\theta}-T_{+-}\right) .
\end{aligned}
$$

Furthermore, this null co-ordinate system characterizes a sphere to be trapped if $\left(\partial_{+} r\right)\left(\partial_{-} r\right)>0$; it is future (or past) trapped if $\partial_{ \pm} r<0$ ( or $>0$ ) with $\partial_{ \pm}$future pointing. However, in the untrapped region $\left(\partial_{+} r\right)\left(\partial_{-} r\right)<0$, the orientation is chosen (locally), so that $\partial_{+}$is outward (i.e., $\left.\partial_{+} r>0\right)$ and $\partial_{-}$ is inward (i.e., $\partial_{-} r<0$ ).

- First law of black hole statics:

In stationary space-time $\exists$, a Killing vector $\xi$ that generates asymptotic time translations and $\xi$ satisfies the Killing equation:

$$
\nabla \otimes \xi^{\natural}=0,
$$

with $\otimes$ denoting the symmetric tensor product. A Killing horizon is a null hypersurface for which the Killing vector is tangent to the null generators, and it may be considered as the boundary of a stationary black hole (or a white hole/cosmological regions). Now, by definition, the vector $\xi \cdot\left(\nabla \wedge \xi^{\natural}\right)$ is parallel to $\xi^{\natural}$ on a Killing horizon, and the proportionality constant is termed as surface gravity, i.e.,

$$
\xi \cdot\left(\nabla \wedge \xi^{\natural}\right)=\kappa \xi^{b}
$$

on a Killing horizon with $\wedge$ denoting the antisymmetric tensor product.

As an example, one may choose the Reissner-Nordström solution of the Einstein-Maxwell equations. The two parameters $e$ and $m$ characterize the charge and asymptotic energy $m$. For $m \geq$ $|e|$, it describes a stationary black hole with surface gravity $\kappa=\frac{\sqrt{m^{2}-e^{2}}}{r^{2}}$ on the Killing horizon: $r=m+\sqrt{m^{2}-e^{2}}$. Therefore, on the horizon, one gets the first law of black hole statics:

$$
\mathrm{d} m=\kappa \frac{\mathrm{d} A}{8 \pi}+\frac{e \mathrm{~d} e}{r},
$$

with ' $\mathrm{d}$ ' the differential in the state space $(m, \sigma)$. Due to the ' $m$ ' term, the above first law cannot be generalized to space-times that are not asymptotically flat. By introducing the quasi-local energy $E$ for the present Reissner-Nordström solution as:

$$
E=m-\frac{e^{2}}{2 r}
$$

the above first law can be rewritten as:

$$
\mathrm{d} E=\frac{\kappa \mathrm{d} A+e^{2} \mathrm{~d} V}{8 \pi} .
$$

This is a physically realistic form of the first law of black hole statics with electromagnetism. In analogy to the internal energy of a black hole, $E$ is the energy on the horizon (rather than at infinity) or, equivalently, $E$ is the energy of the black hole, not the energy of the space-time and is defined as the active gravitational energy.

In black hole dynamics, there is no time-like Killing vector to define surface gravity. Kodama [15] introduced a vector, which for spherically symmetry is defined as: 


$$
K=\operatorname{curl} r
$$

with curl defined on the 2D space normal to the spheres of symmetry. For the double null co-ordinate system, the Kodama vector takes the form:

$$
K=e^{-f_{+-}}\left(\partial_{+} r \partial_{-}-\partial_{-} r \partial_{+}\right) .
$$

The Kodama vector satisfies (i) $k \cdot \nabla r=0$ and (ii) $k \cdot k^{\natural}=\frac{2 E}{r}-1$. The Kodama vector is spatial, null or temporal for trapped, marginal or untrapped spheres, respectively. Therefore, similar to a stationary black hole where the boundary is the hypersurface with the null Killing vector, the horizon of a dynamic black hole is the hypersurface where the Kodama vector is null, i.e., the trapping horizon. Thus, it is possible to have a preferred flow of time for a general spherically-symmetric space-time by the Kodama vector (which coincides with the Killing vector for stationary space-time), and active gravitational energy is the conserved charge associated with the Kodama vector; consequently, it is speculated that dynamic gravitational entropy might be defined in analogy to the method of Wald [16].

Now, it is natural to define dynamic surface gravity by replacing the Killing horizon with the trapping horizon and the Killing vector by the Kodama vector. Therefore, the surface gravity is defined as:

$$
K \cdot\left(\nabla \cdot k^{\#}\right)=\kappa \nabla r
$$

with

$$
K \cdot\left(\nabla \wedge k^{\#}\right)= \pm \kappa k^{\#}
$$

on a trapping horizon $\partial_{ \pm} r=0$ or equivalently a purely geometrical definition:

$$
\kappa=\frac{1}{2} \operatorname{div} \operatorname{grad} r
$$

where the divergence and gradient are taken over the normal 2D plane of the spheres of symmetry. Then, one may speculate that a dynamic spherically-symmetric quantum field has a local Hawking temperature $\frac{h \kappa}{2 \pi}$, but it remains an open question.

Subsequently, in the context of black hole dynamics with the above generalized definition of surface gravity, the first law of black hole dynamics can be written as:

$$
E^{\prime}=\frac{\kappa A^{\prime}}{8 \pi}+w V^{\prime}
$$

where prime stands for differentiation along the trapping horizon: $f^{\prime}=z \cdot \nabla f$ with $z$ a tangent vector to the trapping horizon. Here, the first term on the r.h.s. is the usual term for the black hole dynamics, while the second term denotes the work done along the tangent to the trapping horizon.

In black hole statics, the zeroth law for a stationary black hole states that the surface gravity $\kappa$ is constant. In black hole dynamics, the thermal equilibrium is characterized by the constancy of:

$$
\beta=\frac{\partial S}{\partial E},
$$

where $S$ is the entropy of the system. Therefore, gravithermal equilibrium is defined as the thermal equilibrium in a stationary space-time, and a weak type unified zeroth law is defined as both $\kappa$ and $\beta$ are constants.

\section{Universal Thermodynamics in the FLRW Model}

In universal thermodynamics, it is interesting to study the FLRW space-time from the point of view of a dynamical black hole. It is found that for this homogeneous and isotropic space-time, 
there is no outer trapping horizon; rather, there is an inner trapping horizon, which coincides with the apparent horizon [10-13,17-19]. It is found [17] that the Clausius relation can be obtained by projecting the UFL along the tangent to the trapping horizon. Due to absence of the time-like Killing vector field for non-stationary models, one can introduce the divergence-free Kodama vector [15] field for any time-dependent spherically-symmetric space-time. This Kodama vector acts as an asymptotically time-like Killing vector field. Furthermore, there is a conserved current associated with this vector field $[15,20]$. In the following, the universal thermodynamics for the FLRW model will be discussed in the context of the Kodama vector [21].

We start with the homogeneous and isotropic four-dimensional non-stationary FLRW space-time:

$$
\mathrm{d} s^{2}=-\mathrm{d} t^{2}+\frac{a^{2}(t)}{1-k r^{2}} \mathrm{~d} r^{2}+R^{2}\left(\mathrm{~d} \theta^{2}+\sin ^{2} \theta \mathrm{d} \phi^{2}\right),
$$

where $a(t)$ is the scale factor of the expanding universe, $k$ is the curvature and $R=a(t) r$ is the area radius. This metric can also be written as:

$$
\mathrm{d} s^{2}=h_{i j} \mathrm{~d} x^{i} \mathrm{~d} x^{j}+R^{2}\left(\mathrm{~d} \theta^{2}+\sin ^{2} \theta \mathrm{d} \phi^{2}\right),
$$

where $h_{00}=-1, h_{11}=\frac{a^{2}(t)}{1-k r^{2}}, h_{01}=h_{10}=0, x^{0}=t, x^{1}=r, i, j=1,2$. We can write this metric in terms of null co-ordinates as:

$$
\mathrm{d} \bar{s}^{2}=-2 \mathrm{~d} l \mathrm{~d} m+\bar{R}^{2}\left(\mathrm{~d} \theta^{2}+\sin ^{2} \theta \mathrm{d} \phi^{2}\right),
$$

where $\bar{R}$ is a function of $l$ and $m$, respectively. Suppose the space-time is time orientable, and we consider the future pointing null vectors $\frac{\partial}{\partial l}, \frac{\partial}{\partial m}$, which are given by:

$$
\begin{gathered}
\frac{\partial}{\partial l}=\frac{\partial}{\partial t}-\frac{\sqrt{1-k r^{2}}}{a(t)} \frac{\partial}{\partial r}, \\
\frac{\partial}{\partial m}=\frac{\partial}{\partial t}+\frac{\sqrt{1-k r^{2}}}{a(t)} \frac{\partial}{\partial r} .
\end{gathered}
$$

The expansion of the geodesic of congruences is given by:

$$
\theta_{l}=\frac{1}{\bar{R}} \frac{\partial \bar{R}}{\partial l}, \theta_{m}=\frac{1}{\bar{R}} \frac{\partial \bar{R}}{\partial m} .
$$

Depending on $\theta_{l}$ and $\theta m$, we can have three kinds of surfaces:

- trapped surface: $\theta_{l} \theta_{m}>0$,

- untrapped surface: $\theta_{l} \theta_{m}<0$,

- marginal surface: $\theta_{l} \theta_{m}=0$,

i.e., for a trapped surface, both null expansions converge. In other words, in a trapped surface, both the incoming and outgoing light rays converge to the surface of the sphere [22]. For an untrapped surface, one of the expansions of the null congruence converges, and one of expansions of null congruence diverges. Explicitly, the incoming light rays converge, and the outgoing light rays diverge. In the case of a marginal surface, at least oneof the expansions of null congruences vanishes. Now, for the trapped surface, two situations may occur: both $\theta_{l}, \theta_{m}>0$ and both $\theta_{l}, \theta_{m}<0$. If both $\theta_{l}, \theta_{m}>0$, i.e., both $\frac{1}{\bar{R}} \frac{\partial \bar{R}}{\partial l}, \frac{1}{\bar{R}} \frac{\partial \bar{R}}{\partial m}>0$, then the surface is called the past trapped surface, and if both $\theta_{l}, \theta_{m}<0$, i.e., both $\frac{1}{\bar{R}} \frac{\partial \bar{R}}{\partial l}, \frac{1}{\bar{R}} \frac{\partial \bar{R}}{\partial m}<0$, then the surface is called the future trapped surface. For a marginal surface with $\theta_{l}=0$, we may have the following cases:

- future: $\theta_{m}<0$,

- past: $\theta_{m}>0$, 
- bifurcating: $\theta_{m}=0$,

- outer: $\frac{\partial \theta_{l}}{\partial m}<0$,

- inner: $\frac{\partial \theta_{l}}{\partial m}>0$,

- degenerate: $\frac{\partial \theta_{l}}{\partial m}=0$.

A hypersurface foliated by a non-degenerate marginal hypersurface is called a trapping horizon. For the marginal surface of the future outer trapping horizon of black holes, we have:

$$
\theta_{l}=0, \theta_{m}<0, \frac{\partial \theta_{l}}{\partial l}<0,
$$

while for the marginal surface of the past outer trapping horizon of white holes, we have:

$$
\theta_{l}=0, \theta_{m}>0, \frac{\partial \theta_{l}}{\partial l}<0 .
$$

For the FLRW model, the horizon is taken in analogy to the trapping horizon of a dynamical black hole. Since gravity is negative, we have to deal with the inner horizon.

The trapping horizon is defined as $\left(\partial_{l} R\right)_{R=R_{T}}=0$, i.e.,

$$
R_{T}=\frac{1}{\sqrt{H^{2}+\frac{k}{a^{2}}}}=R_{A}, \quad k=0, \pm 1
$$

where $R_{A}$ is the geometric radius of the apparent horizon and $H$ is the Hubble parameter. We can write the internal energy inside a sphere of area radius $R$ totally in terms of the area radius, i.e., the internal energy is totally a geometric quantity. Now, the internal energy can be written as [14]:

$$
E=\frac{R}{2 G}\left(1-h^{a b} \partial_{a} R \partial_{b} R\right) .
$$

For the FLRW model, the internal energy can be written as:

$$
E=\frac{R^{3}}{2 G}\left(H^{2}+\frac{k}{a^{2}}\right)=\frac{R^{3}}{2 G R_{A}^{2}},
$$

i.e., for the FLRW model, the internal energy can be written in terms of the radius of the apparent horizon and the area radius. According to Hayward [10-13], the UFL can be written as:

$$
d E=A \psi+W d V,
$$

i.e., the change in internal energy can be written as the sum of the work function and the work done for $\mathrm{d} V$ amount of volume change. Here, $A=4 \pi R^{2}$ is the surface area of a sphere of area radius $R$ and $V=\frac{4}{3} \pi R^{3}$ is the volume of that sphere; and the work density,

$$
W=-\frac{1}{2} T^{i j} h_{i j}
$$

is regarded as the work done by a change of the horizon, while the energy-supply term is given by $[23,24]$ :

$$
\Psi_{i}=T_{i}^{j} \partial_{j} R+W \partial_{i} R,
$$

$\psi=\Psi_{a} d x^{i}$ denotes the flow of energy through the horizon of area radius $R$.

The Kodama vector for the FLRW model is given by $[15,25]$ :

$$
K^{i}=\epsilon^{i j} \nabla_{j} R
$$


where $\epsilon^{a b}$ is the two-dimensional Levi-Civita tensor in the radial temporal plane. For the FLRW model, the Levi-Civita tensor is given by:

$$
\epsilon_{i j}=a(t)(d t)_{i} \wedge(d r)_{j},
$$

and the Kodama vector for the FLRW model is explicitly given by:

$$
K^{i}=\left[-a\left(\frac{\partial}{\partial t}\right)^{i}+H R\left(\frac{\partial}{\partial r}\right)^{i}\right] .
$$

The Kodama vector serves as the time-like Killing vector for dynamical black holes and FLRW space-time (as there is no time-like Killing vector in non-stationary FLRW space-time). In de Sitter space, it is very similar to the Killing vector $\left(\frac{\partial}{\partial t}\right)$.

For the present FLRW model, the work density is given by:

$$
W=\frac{1}{2}(\rho-p),
$$

and the energy supply term is given by:

$$
\psi=\left(\frac{\rho+p}{2}\right)\{-H R d t+a d r\}
$$

The work done for $\mathrm{d} V$ amount of volume change is given by:

$$
W d V=2 \pi R^{2}(\rho-p)\{H R d t+a d r\},
$$

and the energy flow across the surface area $A$ of a sphere of area radius $R$ is given by:

$$
A \psi=2 \pi R^{2}(\rho+p)\{-H R d t+a d r\} .
$$

Therefore, the change in internal energy $E$ is given by:

$$
d E=\frac{1}{2 G R_{A}^{3}}\left[R^{3}\left(3 H R_{A}-2 \dot{R}_{A}\right) d t+3 R^{2} R_{A} a d r\right] .
$$

Now, we shall obtain the Friedmann equation directly from the UFL by projecting it along the Kodama vector.

Projecting the internal energy change along the Kodama vector, we obtain:

$$
\left\langle d E, K^{i}\right\rangle=-\frac{a R^{3} H}{G}\left(\dot{H}-\frac{k}{a^{2}}\right) .
$$

Projecting the heat flow across the area $A$ along the Kodama vector, we obtain:

$$
\left\langle A \psi, K^{i}\right\rangle=4 \pi R^{3} H a(\rho+p),
$$

and:

$$
\left\langle W \mathrm{~d} V, K^{i}\right\rangle=0
$$

Therefore, the projection of UFL along the Kodama vector gives:

$$
\dot{H}-\frac{k}{a^{2}}=-4 \pi G(\rho+p),
$$

i.e., we obtain the second Friedmann equation. 
We shall now show the first Friedmann equation by projecting it along a vector $U^{\mu}$ orthogonal to the Kodama vector in the two-dimensional radial temporal plane. The above-mentioned vector is given by:

$$
U^{\mu}=\left(-\frac{1}{a^{2} R}, \frac{1}{H a^{3} R^{2}}, 0,0\right) .
$$

The vector has the following properties: (i) Depending on the components, the vector may be space-like, null or time-like. (ii) The divergence of this vector vanishes (i.e., $\nabla_{\mu} U^{\mu}=0$ ). Therefore, we can find a current associated with that vector given by $\xi^{\mu}=G^{\mu \nu} U_{\nu}$. From the Bianchi identity, we can say the vector $\xi^{\mu}$ is conserved, i.e., the divergence of the vector vanishes, i.e., in mathematical form:

$$
\nabla_{\mu} \xi^{\mu}=0
$$

Projecting the change in internal energy along the vector $U^{\mu}$ gives:

$$
\left\langle d E, U^{\mu}\right\rangle=\frac{R^{2}}{2 G R_{A}^{3} a^{2}}\left[-\left(3 H R_{A}-2 \dot{R}_{A}\right)+\frac{3 R_{A}}{H R^{2}}\right],
$$

Projecting the heat flow along the vector $U^{\mu}$ gives:

$$
\left\langle A \psi, U^{\mu}\right\rangle=\frac{2 \pi R^{2}}{a^{2}}(\rho+p)\left\{H+\frac{1}{H R^{2}}\right\},
$$

and the projection of the work done by $\mathrm{d} V$ amount of volume along the vector $U^{\mu}$ gives:

$$
\left\langle W d V, U^{\mu}\right\rangle=\frac{2 \pi R^{2}}{a^{2}}(\rho-p)\left\{-H+\frac{1}{H R^{2}}\right\} .
$$

Therefore, projecting the UFL along the vector orthogonal to the Kodama vector gives:

$$
H^{2}+\frac{k}{a^{2}}=\frac{8 \pi G}{3} \rho
$$

- $\quad$ projecting along the vector $\left(\frac{2}{H R^{3}}, 0\right)$ and $\left(0,-\frac{2}{3 R^{2} a}\right)$

Projecting the UFL along the vector $\left(\frac{2}{H R^{3}}, 0\right)$, we obtain:

$$
2 \dot{H}+3 H^{2}+\frac{k}{a^{2}}=-8 \pi p .
$$

Projecting the UFL along the vector $\left(0,-\frac{2}{3 R^{2} a}\right)$, we obtain:

$$
H^{2}+\frac{k}{a^{2}}=\frac{8 \pi G}{3} \rho \text {. }
$$

- $\quad$ Projecting along the vector $U^{\mu}$ and $\bar{U}^{\mu}$

Let us consider two linearly independent vectors $U^{\mu}=\left(V^{0}, V^{1}\right)$ and $\bar{U}^{\mu}=\left(V^{0},-V^{1}\right)$ in FLRW space-time where $V^{0}, V^{1}$ are arbitrary functions of $r$ and $t$ with $V^{1} \neq 0$. We now project the UFL along these two vectors: 


$$
\begin{aligned}
& \left(2 \dot{H}+3 H^{2}+\frac{k}{a^{2}}\right) H R^{3} V^{0}+\left(3 H^{2}+3 \frac{k}{a^{2}}\right) R^{2} a V^{1}=8 \pi R^{2}\left(-p H R V^{0}+\rho a V^{1}\right), \\
& \left(2 \dot{H}+3 H^{2}+\frac{k}{a^{2}}\right) H R^{3} V^{0}+\left(3 H^{2}-3 \frac{k}{a^{2}}\right) R^{2} a V^{1}=8 \pi R^{2}\left(-p H R V^{0}-\rho a V^{1}\right),
\end{aligned}
$$

respectively. Adding Equations (57) and (58), we obtain (55), and subtracting (58) from (57), we obtain (56). These two vectors will be divergence free simultaneously if we take $V^{0}=\phi_{1}(r) a^{-3}$ and $V^{1}=\phi_{2}(t) \frac{\sqrt{1-k r^{2}}}{r^{2}}$ where $\phi_{2}(r)$ and $\phi_{2}(t)$ are arbitrary functions of $r$ and $t$ alone, respectively. As these vectors are divergence free, then we can find two conserved currents $C^{\mu}=G^{\mu \nu} U_{v}$ and $\bar{C}^{\mu}=G^{\mu v} \bar{U}_{v}$ associated with these vectors $U^{v}$ and $\bar{U}^{v}$, respectively, i.e., $\nabla_{\mu} C^{\mu}=0$ and $\nabla_{\mu} \bar{C}^{\mu}=0$. Depending on the components, both of these vectors may be time-like, space-like or null.

\section{Clausius Relation from the UFL}

It has been shown that first law of black hole thermodynamics can be obtained by projecting the UFL along the trapping horizon [10-13,17], i.e.,

$$
\langle A \psi, z\rangle=\frac{\kappa}{8 \pi G}\langle d A, z\rangle,
$$

where $z$ is a vector in the direction tangent to the trapping horizon. We shall now show the validity of the Clausius relation for the event horizon.

$$
R_{E}=a \int_{t}^{\infty} \frac{d t}{a} .
$$

The normal vector to the hypersurface $R=R_{E}$ is given by $n_{a}=(-1, a, 0,0)$. The vector $n_{a}$ is a null vector. This vector is also tangential to the event horizon. Then, we can show that the Clausius relation is not satisfied for the event horizon.

The flow of energy across the surface of the sphere of area radius $R_{E}$ during infinitesimal time $\mathrm{d} t$ is given by:

$$
d Q=4 \pi H R_{E}^{3}(\rho+p) d t .
$$

Using the second Friedmann equation, the above relation can be written as:

$$
d Q=-\frac{H R_{E}^{3}}{G}\left(\dot{H}-\frac{k}{a^{2}}\right) d t=\frac{R_{E}^{3}}{G R_{A}^{3}} \dot{R}_{A} d t .
$$

where we have used $R_{A}=\frac{1}{\sqrt{H^{2}+\frac{k}{a^{2}}}}$.

Now, to derive the Clausius relation, we shall use the generalized Hawking temperature [26]:

$$
T_{E}^{(G)}=\alpha \frac{R_{E}}{2 \pi R_{A}^{2}}
$$

with $\alpha=\frac{\dot{R}_{A} R_{E}}{\dot{R}_{E} R_{A}}$.

Therefore, we have the right-hand side of the Clausius relation as:

$$
T_{E}^{(G)} d S_{E}=\frac{R_{E}^{3}}{G R_{A}^{3}} \dot{R}_{A} d t .
$$

From (62) and (64), we can easily obtain the Clausius relation $\delta Q=T_{E}^{(G)} d S_{E}$ on the event horizon. The present approach to obtain the Clausius relation is a more generalized way, and this can be applied for any horizon. 


\section{A Redefinition of Surface Gravity}

The surface gravity at the trapping horizon for the FLRW model is defined as:

$$
\kappa_{T}=\left.\frac{1}{2 \sqrt{-h}} \partial_{i}\left(\sqrt{-h} h^{i j} \partial_{j} R\right)\right|_{R=R_{T}} .
$$

Here, we assume the above definition of surface gravity, and we shall examine the interrelationship between the UFL and Friedmann equations. For any horizon, the surface gravity is defined as:

$$
\kappa=\frac{1}{2 \sqrt{-h}} \partial_{a}\left(\sqrt{-h} h^{a b} \partial_{b} R\right)
$$

or explicitly, we can write it as:

$$
\kappa=-\left(\frac{R}{R_{A}}\right)^{2}\left(\frac{1-\dot{R}_{A} / 2 H R_{A}}{R}\right) .
$$

We know that the Hawking temperature on the horizon of area radius $R_{h}$ is given by $T=\frac{1}{2 \pi R_{h}}$; the amount of heat flow along this surface of area radius $R_{h}$ is given by $d Q=4 \pi R_{h}^{3}(\rho+p) d t$; and the horizon entropy is $S_{h}=\frac{\pi R_{h}^{2}}{G}$; then, we can see that $d Q \neq T d S$. Therefore, we need to modify the Hawking temperature. In this section, we redefine surface gravity for a local Rindler observer. The Unruh temperature $\left(T=\frac{\hbar f}{2 \pi c k_{B}}\right)$ is proportional to the acceleration [27], and the form is very similar to the Hawking temperature of a black hole. If $A$ is the acceleration of the FLRW model, so in analogy, we take the temperature to be proportional to the acceleration of the model $\left(\hbar=c=k_{B}=1\right)$.

$$
T_{R H}=\frac{A}{2 \pi} .
$$

Here, $A$ is defined as:

$$
A=a_{0} R \frac{\ddot{a}}{a}=a_{0} R\left(\dot{H}+H^{2}\right) .
$$

where we have introduced $a_{0}$ as a dimensionless constant of proportionality and $R$ is introduced to match the dimension on both sides. Using the Friedmann equation, the temperature on the local Rindler causal horizon becomes:

$$
T_{R H}=-\frac{2 a_{0} R G}{3}(\rho+3 p) \text {. }
$$

Here, we introduce a new vector $V^{\mu}$ similar to the Kodama vector:

$$
V^{\mu}=\left(-\frac{3}{R^{3}}, \frac{H}{a R^{2}}, 0,0\right) .
$$

Projecting the change in internal energy along the above-mentioned vector, we obtain:

$$
\left\langle d E, V^{\mu}\right\rangle=4 \pi H(\rho+3 p) .
$$

The right-hand side in the above expression is nothing but $\left\langle T_{R H} d S, V^{\mu}\right\rangle$, i.e., we obtain the Clausius relation:

$$
\text { i.e., } \delta Q=T_{R H} d S \text {. }
$$

where we have taken $a_{0}=\frac{3}{2}$ and $S$ is the Bekenstein entropy. It is very interesting to note that the above-mentioned vector has properties similar to the Kodama vector.

(i) $V^{\mu}$ lies in the 2D $r-t$ plane. The vector is neither parallel, nor orthogonal to the Kodama vector, as well as $U^{\mu}$. (ii) Depending on the components, the norm of the vector may be positive, negative or zero, i.e., the vector may be space-like, time-like or null. (iii) The divergence of the vector 
vanishes. Therefore, we can find a conserved current $C^{\mu}=G^{\mu v} V_{v}$ associated with the vector, i.e., $\nabla_{\mu} C^{\mu}=0$.

\section{Conclusions}

In this paper, we have described different aspects of the UFL for homogeneous and isotropic FLRW space-time. We have derived the Friedmann equation from the UFL in three different ways. Firstly, we have projected the UFL along the Kodama vector, and we have obtained the second Friedmann equation, then projected it along a divergenceless vector perpendicular to Kodama vector in the two-dimensional $r-t$ plane and obtained the first Friedmann equation. In the next method, projecting the UFL along the vectors $\left(\frac{2}{H R^{3}}, 0\right)$ and $\left(0,-\frac{2}{3 R^{2} a}\right)$, we have obtained the Friedmann equation for pressure and density components separately. These two vectors are linearly independent, and they form a basis of the $r-t$ plane. In the final method, we have projected the UFL along the vectors $U^{\mu}=\left(V^{0}, V^{1}\right)$ and $\bar{U}^{\mu}=\left(V^{0},-V^{1}\right)$, which may be divergenceless for certain choices and obtained from the Friedmann equations in a combined form. It is also interesting to note that these two vectors form a basis of the two-dimensional $r-t$ plane for $V^{1} \neq 0$.

We have also shown the validity of the Hayward-Kodama definition of surface gravity (65) for dynamical FLRW space-time. Assuming the generalized Hawking temperature, we can also obtain the Clausius relation from the UFL. Finally, we have redefined the surface gravity as a quantity proportional to the acceleration of FLRW space-time, and we can obtain the Clausius relation by projecting the UFL along the divergence-free vector $V^{\mu}$. In the future, we shall generalize the work to more generalized space-time models.

Author Contributions: Both authors contributed equally to this work.

Acknowledgments: The authors are thankful to the anonymous referees for their illuminating comments. S.B. is supported by the CSIRSenior Research Fellowship (File No: 09/096(0843)2015-EMR-I). S.C. acknowledges the financial support of the SERB MATRICS scheme (File No: MTR/2017/000407).

Conflicts of Interest: The authors declare no conflicts of interest.

\section{References}

1. Hawking, S.H.; Ellis, G.F.R. The Large Scale Structure of Space-Time; Cambridge University Press: Cambridge, UK, 1973.

2. Hawking, S.W. Particle Creation by Black Holes. Commun. Math. Phys. 1975, 43, 199-220. [CrossRef]

3. Bekenstein, J.D. Black holes and entropy. Phys. Rev. D 1973, 7, 2333. [CrossRef]

4. Bardeen, J.M.; Carter, B.; Hawking, S.W. The Four laws of black hole mechanics. Commun. Math. Phys. 1973, 31, 161-170. [CrossRef]

5. Jacobson, T. Thermodynamics of space-time: The Einstein equation of state. Phys. Rev. Lett. 1995, 75, 1260. [CrossRef] [PubMed]

6. Padmanabhan, T. Classical and quantum thermodynamics of horizons in spherically symmetric space-times. Class. Quant. Grav. 2002, 19, 5387. [CrossRef]

7. Poisson, E. A Relativist's Toolkit: The Mathematics of Black-Hole Mechanics; Cambridge University Press: Cambridge, UK, 2004.

8. Raychaudhuri, A. Relativistic cosmology. 1. Phys. Rev. 1955, 98, 1123. [CrossRef]

9. Raychaudhuri, A. Relativistic and Newtonian Cosmology. Zeitschrift für Astrophysik 1957, 43, 161-164.

10. Hayward, S.A. Unified first law of black hole dynamics and relativistic thermodynamics. Class. Quant. Grav. 1998, 15, 3147. [CrossRef]

11. Hayward, S.A. Gravitational energy in spherical symmetry. Phys. Rev. D 1996, 53, 1938. [CrossRef]

12. Hayward, S.A. General laws of black hole dynamics. Phys. Rev. D 1994, 49, 6467. [CrossRef]

13. Hayward, S.A.; Mukohyama, S.; Ashworth, M.C. Dynamic black hole entropy. Phys. Lett. A 1999, 256, 347-350. [CrossRef]

14. Misner, C.W.; Sharp, D.H. Relativistic equations for adiabatic, spherically symmetric gravitational collapse. Phys. Rev. 1964, 136, B571. [CrossRef] 
15. Kodama, H. Conserved Energy Flux for the Spherically Symmetric System and the Back Reaction Problem in the Black Hole Evaporation. Prog. Theor. Phys. 1980, 63, 1217-1228. [CrossRef]

16. Wald, R.M. Black hole entropy is the Noether charge. Phys. Rev. D 1993, 48, R3427. [CrossRef]

17. Cai, R.G.; Cao, L.M. Unified first law and thermodynamics of apparent horizon in FRW universe. Phys. Rev. D 2007, 75, 064008. [CrossRef]

18. Bak, D.; Rey, S.J. Cosmic holography. Class. Quant. Grav. 2000, 17, L83. [CrossRef]

19. Akbar, M.; Cai, R.G. Thermodynamic Behavior of Friedmann Equations at Apparent Horizon of FRW Universe. Phys. Rev. D 2007, 75, 084003. [CrossRef]

20. Hayward, S.A. Quasilocal gravitational energy. Phys. Rev. D 1994, 49, 831. [CrossRef]

21. Haldar, S.; Bhattacharjee, S.; Chakraborty, S. Unified first law and some general prescription: A redefinition of surface gravity. Eur. Phys. J. C 2017, 77, 604. [CrossRef]

22. Paetz, T.T.; Simon, W. Marginally outer trapped surfaces in higher dimensions. Class. Quant. Grav. 2013, 30, 235005.

23. Cai, R.G.; Cao, L.M.; Hu, Y.P. Hawking Radiation of Apparent Horizon in a FRW Universe. Class. Quant. Grav. 2009, 26, 155018. [CrossRef]

24. Cai, R.G.; Kim, S.P. First law of thermodynamics and Friedmann equations of Friedmann-Robertson-Walker universe. J. High Energy Phys. 2005, 2005, 050. [CrossRef]

25. Abreu, G.; Visser, M. Kodama time: Geometrically preferred foliations of spherically symmetric space-times. Phys. Rev. D 2010, 82, 044027. [CrossRef]

26. Chakraborty, S. Generalized Bekenstein-Hawking system: Logarithmic Correction. Eur. Phys. J. C 2014, 74, 2876. [CrossRef]

27. Misner, C.W.; Thorne, K.S.; Wheeler, J.A. Gravitation; W. H. Freeman and Company: San Francisco, CA, USA, 1973.

(C) 2018 by the authors. Licensee MDPI, Basel, Switzerland. This article is an open access article distributed under the terms and conditions of the Creative Commons Attribution (CC BY) license (http:/ / creativecommons.org/licenses/by/4.0/). 